\title{
Encapsulating Peritoneal Sclerosis: A Rare Devastating Peritoneal Disease: Case Series and Literature Review
}

\author{
Muftah Othman ${ }^{1}$, Hassan Al Malki1, Fadwa Al Ali1, Hanan Farghaly², Muhammed Asim¹, \\ Omar Fituri ${ }^{1}$
}

${ }^{1}$ Nephrology-Medicine Department, Hamad Medical Corporation, Doha, Qatar

${ }^{2}$ Pathology Department, Hamad Medical Corporation, Doha, Qatar

Email: *mothman5@hamad.qa

How to cite this paper: Othman, M., Al Malki, H., Al Ali, F., Farghaly, H., Asim, M. and Fituri, O. (2019) Encapsulating Peritoneal Sclerosis: A Rare Devastating Peritoneal Disease: Case Series and Literature Review. Open Journal of Nephrology, 9, 115-126. https://doi.org/10.4236/ojneph.2019.94012

Received: September 22, 2019 Accepted: November 2, 2019 Published: November 5, 2019

Copyright $\odot 2019$ by author(s) and Scientific Research Publishing Inc. This work is licensed under the Creative Commons Attribution International License (CC BY 4.0).

http://creativecommons.org/licenses/by/4.0/

cc) (i) Open Access

\begin{abstract}
Background: Encapsulating peritoneal sclerosis is a rare but life-threatening condition in peritoneal dialysis. It is associated with extensive thickening and fibrosis of the peritoneum resulting in recurrent small bowel obstructions and malnutrition. Herein we present case series of encapsulating peritoneal sclerosis diagnosed and treated in a large center of peritoneal dialysis in the State of Qatar followed by literature review to increase the awareness of physicians to it. Case Presentation: The data were collected retrospectively from 180 peritoneal dialysis patients at Hamad General Hospital, Doha, Qatar, between 2008 and 2016. The diagnosis of encapsulating peritoneal sclerosis was based on clinical and radiological features. Seven patients (3.8\%) were diagnosed with encapsulating peritoneal sclerosis. The mean age of the patients was 49 years (range, 23 to 65 years). The mean duration of peritoneal dialysis was 6 years (range, 3 to 7 years). The mean peritonitis episodes were 1.8 (range 1 to 4 episodes). Five patients were diagnosed with encapsulating peritoneal sclerosis after stopping peritoneal dialysis, and two patients diagnosed while they were on peritoneal dialysis treatment. Five patients are still alive and were treated with oral prednisolone and tamoxifen. Two patients underwent adhesiolysis surgery, one of them died due to recurrent sepsis and malnutrition. Another patient died because of cardiac arrest during hemodialysis. The overall mortality rate was $28.5 \%$ in our series. Conclusions: Encapsulating peritoneal sclerosis is a rare and life-threatening peritoneal disease in chronic peritoneal dialysis patients. It requires high index of suspicion for early diagnosis and treatment.
\end{abstract}

\section{Keywords}

Encapsulating Peritoneal Sclerosis, Peritoneal Dialysis, Bowel Obstruction 


\section{Background}

Encapsulating Peritoneal Sclerosis (EPS) is a rare but a serious complication of peritoneal dialysis (PD). It is associated with significant morbidity and mortality. It may present even after PD has been discontinued. EPS is characterized by thickening and fibrosis of the peritoneal membrane with formation of a fibrous cocoon that encapsulates the bowel and complicated by intestinal obstruction, malnutrition, weight loss, bowel ischemia, infection and death.

EPS is often missed in its early stages and requires a high index of suspicion to diagnose [1]. According to the International Society for Peritoneal Dialysis (ISPD) definition, the diagnosis of EPS requires both:

1) Clinical features of persistent or recurrent intestinal obstruction.

2) Radiological or pathological evidence of peritoneal membrane thickening and bowel encapsulation.

It is noted that, EPS is not exclusive to PD; it has been associated with other conditions, namely: systemic autoimmune disease, abdominal malignancies and surgeries, peritoneal tuberculosis and the use of intraperitoneal disinfectant for peritoneal lavage [2].

The incidence of EPS varies between countries from $0.7 \%$ to $3.3 \%$. A higher rate of EPS is reported as patients remain on $\mathrm{PD}$ for a longer time with increased awareness of this complication. The mortality of patients with EPS varies between $26 \%$ and $57 \%$ [1]. Herein we present case series of EPS diagnosed and treated in a large center of peritoneal dialysis in the State of Qatar followed by literature review to increase the awareness of nephrologists, radiologists, pathologist and surgeons of this rare and fatal complication of long-term peritoneal dialysis therapy.

\section{Case Presentation}

\subsection{Methods}

The data were collected from the medical records at Hamad General Hospital, Doha, Qatar between 2008 and 2016. EPS diagnosis was made based on clinical and radiological features according to the International Society of Peritoneal Dialysis guideline (ISPD). Only seven cases of EPS were diagnosed at our hospital between 2008 and 2016. Demographic and clinical data including age, gender, duration of PD, primary causes of end-stage renal disease, number of peritonitis episodes, treatment options and outcomes were retrieved.

\subsection{Demographic and Clinical Characteristics of the Case Series}

As shown in Table 1, seven patients (3.8\%) were diagnosed with EPS among 180 PD patients during 8-years study period. It comprised of six males and one female patient. The mean age of the patients at the time of diagnosis was 49 years (range, 23 to 65 years). ESRD was due to diabetic nephropathy in 4 patients while adult polycystic kidney disease, hypertensive nephrosclerosis and rapidly progressive glomerulonephritis were the causes in the other 3 patients. The 
Table 1. Clinico-Demographic Characteristics of EPS Case Series.

\begin{tabular}{|c|c|c|c|c|c|c|}
\hline Case & Age/sex & $\begin{array}{l}\text { Primary } \\
\text { diagnosis }\end{array}$ & $\begin{array}{l}\text { PD duration } \\
\text { (year) }\end{array}$ & $\begin{array}{l}\text { Peritonitis } \\
\text { episodes }\end{array}$ & Treatment & Outcome \\
\hline 1 & $65 / \mathrm{M}$ & DN & 6 & 4 & $\begin{array}{c}\text { Adhesiolysis } \\
\text { Prednisolone } 40 \mathrm{mg} \\
\text { Tamoxifen } 10 \mathrm{mg} \text { bid }\end{array}$ & Alive \\
\hline 2 & $60 / \mathrm{M}$ & $\mathrm{DN}$ & 6 & 4 & $\begin{array}{l}\text { Prednisolone } 40 \mathrm{mg} \\
\text { Tamoxifen } 10 \mathrm{mg} \text { bid }\end{array}$ & Alive \\
\hline 3 & $65 / \mathrm{M}$ & ADPKD & 3 & 1 & None & Died \\
\hline 4 & $23 / \mathrm{F}$ & RPGN & 7 & 1 & $\begin{array}{l}\text { Prednisolone } 40 \mathrm{mg} \\
\text { Tamoxifen } 10 \mathrm{mg} \text { bid }\end{array}$ & Alive \\
\hline 5 & $42 / \mathrm{M}$ & HTN & 6 & 1 & $\begin{array}{l}\text { Prednisolone } 40 \mathrm{mg} \\
\text { Tamoxifen } 10 \mathrm{mg} \text { bid }\end{array}$ & Alive \\
\hline 6 & $40 / \mathrm{M}$ & $\mathrm{DN}$ & 7 & 1 & $\begin{array}{c}\text { Adhesiolysis } \\
\text { TPN }\end{array}$ & Died \\
\hline 7 & $50 / \mathrm{M}$ & $\mathrm{DN}$ & 7 & 1 & $\begin{array}{l}\text { Prednisolone } 40 \mathrm{mg} \\
\text { Tamoxifen } 10 \mathrm{mg} \text { bid }\end{array}$ & Alive \\
\hline
\end{tabular}

$\mathrm{M}=$ male, $\mathrm{F}=$ female, $\mathrm{DN}=$ Diabetic nephropathy, $\mathrm{ADPKD}=$ adult polycystic kidney disease, $\mathrm{RPGN}=\mathrm{ra}-$ pidly progressive glomerulonephritis, $\mathrm{HTN}=$ Hypertensive nephrosclerosis. TPN $=$ total parental nutrition.

mean duration of PD was 6 years (range, 3 to 7 years). The mean peritonitis episodes were 1.8 (range, 1 to 4 episodes). Five patients were diagnosed with EPS after stopping $\mathrm{PD}$, and two patients diagnosed while they were on PD treatment. All patients were treated with icodextrin solution at diagnosis. Five patients are still alive and were treated with oral prednisolone and tamoxifen. Total parenteral nutrition was used in one patient. Two patients underwent adhesiolysis surgery. One of them died due to recurrent sepsis and malnutrition. Another patient died because of cardiac arrest during hemodialysis. The time to death of the two patients from diagnosis of EPS was 12 and 16 months respectively. The overall mortality rate was $28.5 \%$ in our series.

\subsection{Diagnosis and Treatment of Our EPS Case Series}

Case 1: Almost one year after cessation of PD due to refractory peritonitis, he was presented with abdominal pain and distension. Abdominal X-ray showed multiple air fluid levels suggestive of intestinal obstruction. His abdominal ultrasound showed multiloculated collection $(6 \times 5 \times 4 \mathrm{~cm})$. He had a CT scan of the abdomen that showed a pocket of fluid $(8 \times 7 \times 5 \mathrm{~cm})$ in the right iliac fossa with dilated small bowel loops and air fluid levels suggestive of intestinal obstruction. In addition, it showed changes of encapsulation, loculated fluid collection in the right iliac fossa and peritoneal membrane enhancement (Figure 1). The diagnosis of EPS was made based on clinical and radiologic features.

He underwent laparotomy to relief his intestinal obstruction. The interaoperative findings showed dense fibrous adhesions with membrane encapsulating the small bowel loops and abscess cavities noted in the right iliac fossa and pelvis. Adhesiolysis of extensive small bowel adhesions and excision of the membrane 


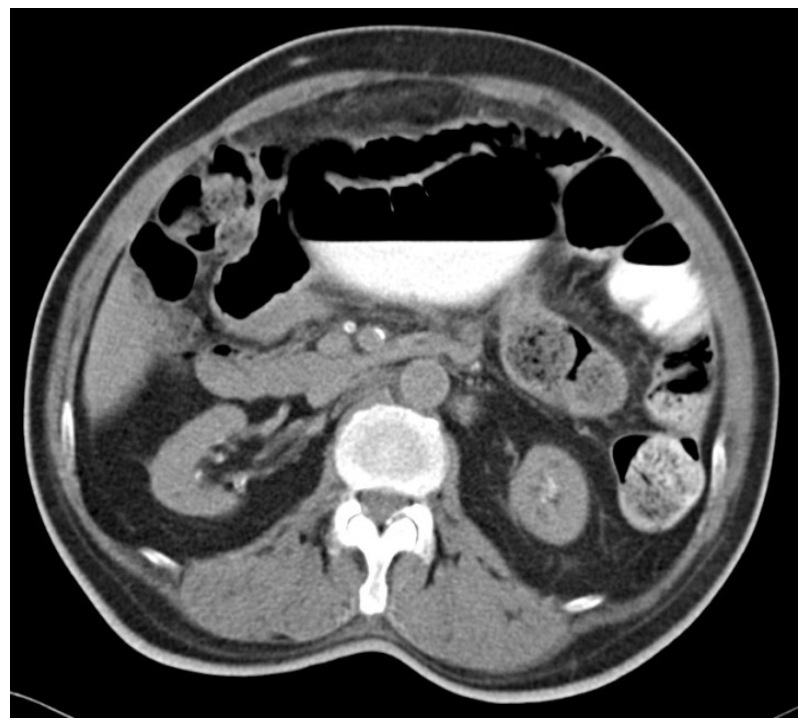

Figure 1. CT scan abdomen showing dilated small bowel loops with air fluid levels and clumping of bowel in central abdomen with encapsulation.

enclosing the adherent bowels were made. Histopathological examination of the surgical specimen of the peritoneum revealed denuded mesothelial fibroadipose tissue, dense fibrous and granulation tissues and significant vascular proliferation (Figure 2). He was started on prednisolone $40 \mathrm{mg}$ daily and tamoxifen 10 $\mathrm{mg}$ twice daily. He was discharged with stable conditions and now he is alive and asymptomatic for 6 years after diagnosis of EPS.

Case 2: One year after switching him to hemodialysis (HD) due to multiple peritonitis episodes and inadequate $\mathrm{PD}$, he was presented with recurrent abdominal pain, vomiting, abdominal distension and weight loss. The patient had a CT scan of the abdomen that showed loculated fluid collection and enhancing bowel wall (Figure 3). Abdominal CT scan guided aspiration showed old dark blood. He was diagnosed with EPS and treated with prednisolone $40 \mathrm{mg}$ daily and tamoxifen $10 \mathrm{mg}$ twice daily. Currently, he is doing fine on HD for 8 years after diagnosis of EPS.

Case 3: He was treated with PD for 3 years due to the failure of his kidney transplant for his adult polycystic kidney disease. He then switched to HD due to refractory peritonitis. Two months after switching to HD, he started to have recurrent abdominal pain and ascites requiring repeated CT guided aspiration. His abdominal CT scan showed dilated bowel loops with air fluid level, thickened bowel wall, and loculated collection consistent with EPS. However, he died because of cardiac arrest during hemodialysis before starting treatment of EPS.

Case 4: She was started on PD for ESRD due to rapidly progressive glomerulonephritis and she remained on PD for 7 years. She had recurrent blood tinged PD effluent which was initially presumed due to menstruation. Additionally, she had weight loss of about $10 \mathrm{~kg}$ over 6 months. She had one peritonitis episode. Her X-ray of the abdomen demonstrated calcification of small bowel and an abdominal CT scan revealed moderate ascites with diffuse calcification along the 

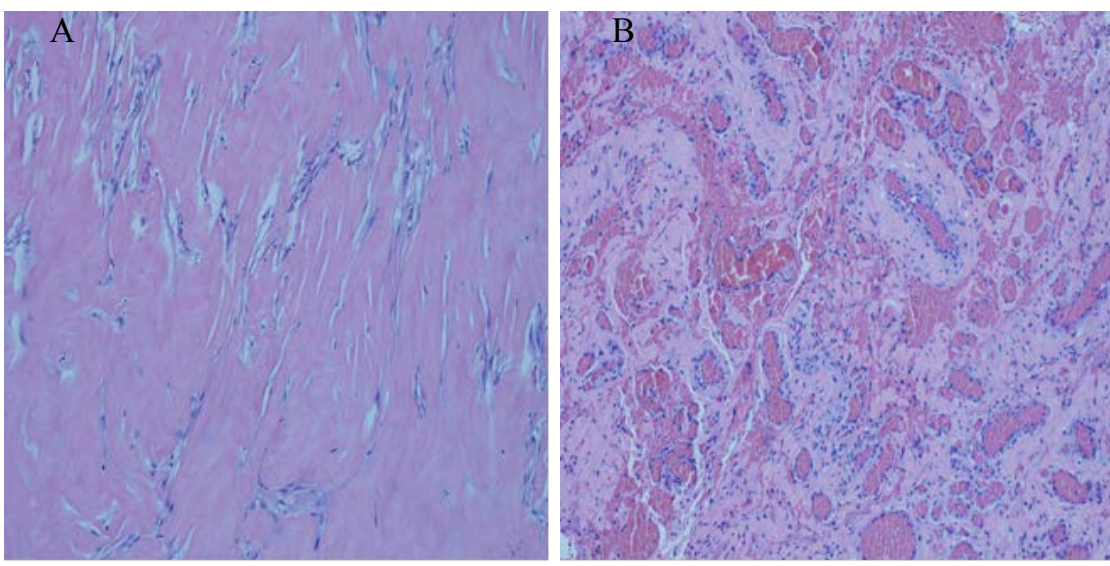

Figure 2. Peritoneal biopsy of the case number 1: (A) Section of the encapsulating peritoneal sclerosis demonstrated abundant dense thick hyalinized fibrosis infiltrating the tissue, obvious fibroblasts proliferation, moderate chronic inflammation and absence of encapsulation (hematoxylin and eosin (H\&E) stain, 20x). (B) Significant vascular proliferation, consistent with capillary angiogenesis associated with chronic inflammation (hematoxylin and eosin (H\&E) stain, 10x).

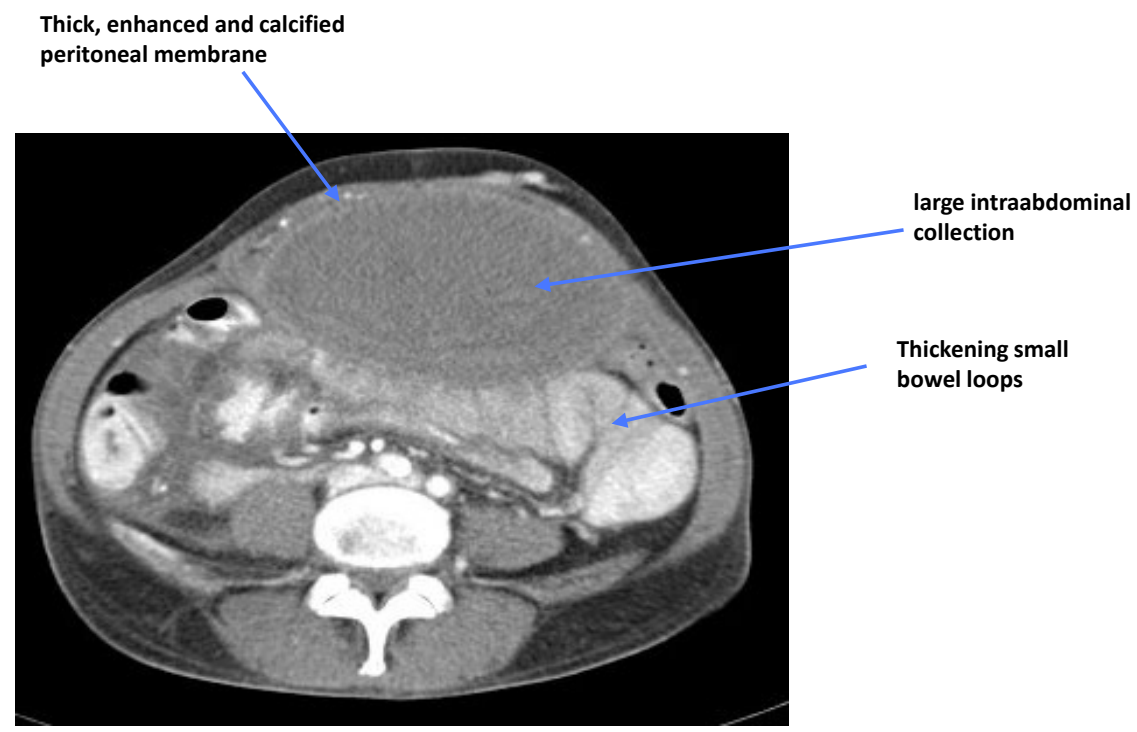

Figure 3. CT scan abdomen showing large intraabdominal collection with peritoneal wall thick, enhanced and calcified peritoneal membrane. Small bowel loops are thick walled and gathered together.

parietal peritoneum and small bowel with multiple non-dilated small bowel loops clumped in the central abdomen. The patient was diagnosed with EPS during PD then shifted to HD. She was started on prednisolone $40 \mathrm{mg}$ daily and tamoxifen $10 \mathrm{mg}$ twice daily. Right now, she is doing well on hemodialysis for 5 years after diagnosis of EPS.

Case 5: He was on PD for 6 years then shifted to HD because of refractory peritonitis to treatment. The patient was presented 2 months later with fever, abdominal pain, constipation and marked weight loss from $125 \mathrm{~kg}$ to $83 \mathrm{~kg}$ over 6 months. He also had resistant anemia to erythropoietin associated with very 
high ESR and CRP and hypoalbuminemia of $20 \mathrm{gm} / \mathrm{L}$. He had a CT scan of the abdomen that revealed large loculated intraperitoneal fluid with thin enhancing septa in the collection and thickened peritoneum. Fluid aspiration from the collection was bloody and negative for tuberculosis and malignancy. He was diagnosed with EPS and started on prednisolone $40 \mathrm{mg}$ daily and tamoxifen $10 \mathrm{mg}$ twice daily. Now he is alive and doing well on hemodialysis for 5 years after diagnosis of EPS.

Case 6: The patient remained on PD for 7 years. He developed peritonitis with septic shock and therefore, was shifted temporary to HD. Then PD catheter was reinserted and continued on PD for another 1 year. During that year, he started to have anorexia, abdominal pain, muscle wasting and weight loss (30 $\mathrm{kg}$ ). Also, he was noted to have fever with negative blood cultures. He had malnutrition evidenced by severe hypoalbuminemia, hypophosphatemia and anemia. Abdominal CT scan demonstrated loculated fluid collection $(13 \times 5 \mathrm{~cm})$ with multiple dilated small bowel loops and wall thickness. The patient was diagnosed with EPS and therefore treated with total parenteral nutrition and shifted to HD due to inadequate PD and poor ultrafiltration. He underwent laparotomy for intestinal obstruction that was complicated by multiple perforation and sepsis. Few months later, he had ileal perforation and underwent resection and anastomosis. However, he died one year later with malnutrition and recurrent sepsis.

Case 7: After the failure of his kidney transplant, the patient was treated with PD for 7 years. He was switched to HD due to recurrent peritonitis and ultrafiltration failure. Three months later, he started to have abdominal distension but no constipation, vomiting nor fever. His abdominal CT scan unveiled a well-defined collection $(18 \times 11 \times 7 \mathrm{~cm})$ connected with another collection in the left iliac fossa $(16 \times 11 \times 8 \mathrm{~cm})$ with enhanced bowel wall loops suggestive of EPS. The patient was started on prednisolone $40 \mathrm{mg}$ daily and tamoxifen $10 \mathrm{mg}$ twice daily. Currently, he is asymptomatic on HD for 4 years after diagnosis of EPS.

\section{Discussion}

The PD program was started at Hamad General Hospital, Doha, Qatar in 1996 with two cases that was increased to around 80 cases in 2008. Now we have 180 patients on peritoneal dialysis which make our center as the largest in the region. During this period, we diagnosed seven cases of EPS with a rate of 3.8\%. Most of our cases of EPS developed after 3 to 7 years of PD treatment.

The incidence of EPS increases with increasing duration of peritoneal dialysis therapy. The incidence of EPS in a study from Japan was ranging from $0 \%$ at 3 years, $5.8 \%$ at 10 years and $17.2 \%$ with $100 \%$ mortality in patients on PD for over 15 years [2].

In a study from Scotland of 46 cases of EPS out of 1238 patients on PD showed an 8-year cumulative incidence of EPS of $8.1 \%$ with the median PD duration of 5.1 years. In this study 12 patients (26\%) were on PD at diagnosis and 
33 patients (72\%) were diagnosed within 2 years after PD was stopped [1].

Risk factors of EPS are many, including: longer duration on PD and paradoxically discontinuation of $\mathrm{PD}$, use of hypertonic glucose dialysate and frequent peritonitis episodes. Other risk factors are ultrafiltration failure, absence of residual renal function and severe peritonitis (e.g Staph aureus, Pseudomonas, fungal). Younger patients develop EPS more than older patients because they are more likely to remain longer on PD [1].

In addition, there are few reports of an increase in EPS following kidney transplantation [3] [4]. EPS develops typically within 1 year after kidney transplantation. Two reasons have been proposed for that: firstly, treatment with calcineurin inhibitors (CNI) after kidney transplantation is associated with a decreased need for steroids which has a protective effect on the development of EPS. Secondly, CNIs have profibrotic properties that upregulate expression of TGF- $\beta$ and might also promote the development of EPS [4].

\section{Pathogenesis of EPS:}

The pathogenesis of EPS is unclear; however, it can be explained by a two-hit mechanism. The first hit with long-term exposure of peritoneal membrane to uremia, glucose degradation products and $\mathrm{PD}$ fluid with its low $\mathrm{pH}$ and high glucose concentration leads to a chronic peritoneal membrane injury and mesothelial disruption. It triggers a cascade of fibrotic process resulting in, activation of myofibroblasts and increased deposition of extracellular matrix, a condition called simple sclerosis. Genetic predisposition, frequent episodes of peritonitis, and abrupt discontinuation of PD play as a second hit that leads to development of peritoneal membrane fibrosis, bowel adhesion and encapsulation [5].

Diagnosis of EPS:

The diagnosis of EPS relies on clinical, radiological features and pathological findings.

Clinical features of EPS:

There are four proposed stages of EPS according to Nakamoto et al. 2005:

The pre-EPS or asymptomatic stage 1 is characterized clinically by ultrafiltration failure and progression to fast transport status and pathologically with no inflammation, mesothelial denudation, vasculopathy, peritoneal thickening, fibrosis, and peritoneal calcifications.

The inflammatory stage 2 can be missed or misdiagnosed and it precedes the fibrosing phase. It is characterized by an increase in inflammatory markers with high CRP, leukocytosis, resistant anemia, fever, anorexia, weight loss, malnutrition, hypoalbuminemia and bloody dialysate with hemorrhagic ascites. Pathologically, it shows evidence of inflammation, mononuclear cells and fibrin degradation products.

The encapsulating or progressive stage 3 is characterized by the disappearance of signs of inflammation and appearance of symptoms and signs of ileus including nausea, vomiting, abdominal pain, constipation, abdominal mass and ascites. Pathologically, there are adhesions and progressive encapsulation.

The ileus or obstructive stage 4 is characterized by a complete ileus with re- 
current intestinal obstruction, anorexia, progressive malnutrition and abdominal mass. Pathological findings include lack of inflammation and complete bowel obstruction and/or encapsulating fibrous cocoon [6].

\section{Radiological features of EPS:}

CT scanning is the investigation of choice and the best-studied imaging modality in patients with established EPS and it helps monitor disease progression. Unfortunately, the CT findings are not adequately reliable to enable diagnosis of early stages of EPS (Pre-symptomatic and Inflammatory stages). The most specific features on CT scan are bowel tethering and peritoneal calcification. Basically, MRI is not helpful since it is poor at assessing peritoneal calcification and there are concerns about nephrogenic systemic fibrosis from gadolinium in dialysis patients. According to a study by Vlijm and colleagues, the CT scan findings of EPS are: peritoneal thickening, peritoneal calcification, peritoneal enhancement, fluid loculation and septation, adhesions of bowel loops, and signs of bowel obstruction. Three out of the six items or two of the five items if no intravenous contrast medium is administered (i.e. no peritoneal enhancement) gave a sensitivity of $79 \%-100 \%$; and a specificity of $88 \%-94 \%$ [7].

\section{Pathologic Findings of EPS:}

Typical macroscopic appearance is observed at laparotomy or laparoscopy.

Pathologically, EPS can range from a thin membrane on the visceral and/or parietal peritoneum to a cocoon-like encapsulation of the entire intestine. Variable degrees of peritoneal thickening and calcification may be seen. Fibrous bands can form between loops of bowel, and small loculated abscesses may also be seen due to local perforation. Histological findings are characteristic but not specific for EPS because it overlaps with peritoneal membrane changes that occur in long term PD (e.g simple sclerosis). Significant histological findings in patients with EPS are: thickening of the sub-mesothelial cell layer, vasculopathy, arterial occlusion, inflammation, tissue and arterial calcification, and ossification [2]. It is important to obtain peritoneal biopsy and histology if diagnosis is doubtful. Differential diagnosis of EPS includes tuberculous peritonitis, peritoneal mesothelioma and carcinomatosis, and post-transplant small bowel lymphoma.

\section{Early Diagnosis \& Prediction of EPS:}

Although all patients with EPS have ultrafiltration failure, only $50 \%$ of patients who continue PD for 3 years after development of ultrafiltration failure will develop EPS.

So is it possible to identify and predict those at risk of developing EPS?

1) Routine CT scanning: Unfortunately, the CT scan findings are not sufficiently reliable to diagnose early stages of EPS (Pre-symptomatic and Inflammatory stages).

2) Serial peritoneal equilibration (PET) test on regular basis to detect ultrafiltration failure and high transport status that at risk of EPS is still premature. Sampimon and colleagues compared 12 EPS patients versus 21 patients with ultrafiltration failure and 26 patients with normal ultrafiltration with yearly peritoneal equilibration test (PET). They found no difference in either solute trans- 
port rate or ultrafiltration volume in patients with ultrafiltration failure with and without EPS [8]. Lambie and Davies studied 9 patients with EPS versus 36 PD control patients. They found that EPS patients had lower ultrafiltration volumes for at least 2 years before the diagnosis of EPS. So they concluded that serial PET may allow for early identification of high-risk patients to develop EPS. However, this study was too small to define the cut-off ultrafiltration volumes for different solute transport rates to be used in clinical practice [9].

3) Measurement of potential biomarkers in peritoneal effluent helps in early diagnosis of EPS. Combination of low dialysate Cancer Antigen (CA125) and a high dialysate Interleukin (IL-6) in patients with ultrafiltration failure gave a sensitivity of $70 \%$ and a specificity of $100 \%$ for early diagnosis of EPS. However, further research is still needed to identify definitive biomarkers for early diagnosis of EPS [10].

\section{Prevention of EPS:}

Preserved residual renal function, minimal peritonitis episodes, reduced exposure of the peritoneal membrane to a high glucose dialysate and the use of biocompatible PD solutions decrease the risk of developing EPS [1]. Some PD programs in Japan suggested the use of peritoneal lavage to prevent EPS in patients with prolonged PD therapy and increased peritoneal membrane permeability and inflammatory markers [11]. In addition, the combination of PD and HD therapy is considered in some centers in Japan to prevent EPS by reducing the peritoneal membrane exposure to PD solutions, minimizing the risk of peritonitis and overcoming the possible increased risk caused by terminating PD. However, no studies with adequate design or proper sample size yet published to allow for an assessment of the role of this combined therapy.

\section{Is there any expiry date for peritoneal dialysis to prevent EPS?}

A Japanese data showed a dramatic increase in the incidence of EPS after 8 years on PD and recommended preemptively to discontinue PD at that stage [2]. However, the UK guideline stated that routine pre-emptive switching to HD after a specified time on PD is not recommended [1]. Because EPS may develop or worsen after stopping PD and there are no prospective data showing any benefit of preemptively switching long term PD patients to HD. Therefore, each patient needs to be considered individually according to the ISPD guidelines [1]. Moreover, Garosi and Oreopoulos resist the concept of expiry date in PD to prevent EPS. This is due to multiple reasons including: low incidence of EPS and most cases being diagnosed after stopping PD or post kidney transplantation. Furthermore, the alternatives after stopping PD are either shifting the patient to HD or death because of uremia [12].

\section{Management of EPS:}

There is no established treatment for EPS; although evidence from small case studies suggests that steroids and tamoxifen may be beneficial. Anti-fibrotic agent tamoxifen is a selective estrogen receptor modulator and is a nonsteroidal antiestrogenic drug that is effective in the treatment of several fibrotic diseases, such as retroperitoneal fibrosis. Immunosuppressive agents including steroids 
are effective in the early stages of EPS by inhibiting the inflammation that precedes encapsulation. However, other agents like mycophenolate mofetil and azathioprine were tried in treatment of EPS but remain weak as compared to steroids. A retrospective analysis of data from the Dutch EPS study showed a decrease in the mortality of patients with EPS treated with tamoxifen compared to the patients not treated with tamoxifen ( $45.8 \%$ vs $74.4 \%$ respectively) [4]. Therefore, a trial of treatment with steroids and/or tamoxifen is recommended when EPS is diagnosed.

Surgical intervention like peritonectomy or partial and complete enterolysis (adhesiolysis) is recommended in the encapsulating stage of EPS to relieve bowel obstruction [13] [14]. The recurrence rates of EPS after surgical intervention may reach $12.5 \%$ and $25 \%$ in Japan and the UK respectively [13]. Tamoxifen and steroids may also be continued post-surgery to prevent recurrence of EPS [13].

The use of nutritional support is essential and should be the mainstay of therapy for patients with EPS until bowel function recovers. Generally, PD should be discontinued and the patient transferred to HD [1]. However, mild cases with limited life expectancy and quality of life should be reconsidered before switching to hemodialysis. Few case reports and small cases series from Japan suggest additional potential therapeutic roles for peritoneal lavage by leaving the catheter in situ [15].

\section{Conclusions}

EPS is a rare and life-threatening complication of PD. Many physicians are unaware of it. The major risk factor for EPS is prolonged PD therapy. It requires high index of suspicion, because some patients may no longer be on PD at the time of diagnosis. Patients may present with abdominal pain, hemorrhagic ascites and bowel obstruction. Characteristic CT scan findings are required to diagnose EPS. Laparotomy or laparoscopy sometimes needed to confirm the diagnosis. Pathologists must be aware of the microscopic features of EPS to ensure accurate diagnosis.

The choice of either medical or surgical treatment is based on the stage of the EPS. Management includes cessation of PD with transfer to HD, sustained bowel rest with nutritional support and trial of steroids and tamoxifen. If conservative therapy fails, surgical therapy must be considered.

\section{Declarations}

\section{Ethics Approval and Consent to Participate}

These case series were approved for publication by the ethics committee of Medical Research Centre at Hamad General Hospital, Doha, Qatar with Ref No: MRC-04-19-117.

\section{Consent for Publication}

Written informed consent was provided by the patients or their next of kin for 
those who died (case 3 and 6) and is available on request.

\section{Availability of Data and Materials}

The clinical data of these case series are stored in the hospital electronic medical records and cannot be shared.

\section{Funding}

None.

\section{Authors' Contributions}

MO collected, analyzed the clinical data and prepared the manuscript. HA, FA, $\mathrm{HF}, \mathrm{MA}$ and OF were involved in the clinical care of these patients and were involved in drafting and revision of the original manuscript. HF prepared and reported the histopathology of peritoneal biopsy. All authors read and approved the final manuscript.

\section{Acknowledgements}

None.

\section{Conflicts of Interest}

The authors declare no conflicts of interest regarding the publication of this paper.

\section{References}

[1] Brown, E.A., Bargman, J., van Biesen, W., et al. (2017) Length of Time on Peritoneal Dialysis and Encapsulating Peritoneal Sclerosis-Position Paper for ISPD: 2017 Update. Peritoneal Dialysis International, 37, 362-374. https://doi.org/10.3747/pdi.2017.00018

[2] Kawaguchi, Y., Saito, A., Kawanishi, H., et al. (2005) Recommendations on the Management of Encapsulating Peritoneal Sclerosis in Japan, 2005: Diagnosis, Predictive Markers, Treatment, and Preventive Measures. Peritoneal Dialysis International, 25, S83-S95.

[3] Yamamoto, R., Otsuka, Y., Nakayama, M., et al. (2005) Risk Factors for Encapsulating Peritoneal Sclerosis in Patients Who Have Experienced Peritoneal Dialysis Treatment. Clinical and Experimental Nephrology, 9, 148-152. https://doi.org/10.1007/s10157-005-0349-8

[4] Korte, M.R., Sampimon, D.E., Lingsma, H.F., et al. (2011) Risk Factors Associated with Encapsulating Peritoneal Sclerosis in Dutch EPS Study. Peritoneal Dialysis International, 31, 269-278. https://doi.org/10.3747/pdi.2010.00167

[5] Augustine, T., Brown, P.W., Davies, S.D., Summers, A.M. and Wilkie, M.E. (2009) Encapsulating Peritoneal Sclerosis: Clinical Significance and Implications. Nephron Clinical Practice, 111, c149-c154. https://doi.org/10.1159/000191214

[6] Nakamoto, H. (2005) Encapsulating Peritoneal Sclerosis-A Clinician's Approach to Diagnosis and Medical Treatment. Peritoneal Dialysis International, 25, S30-S38.

[7] Vlijm, A., Stoker, J., Bipat, S., et al. (2009) Computed Tomographic Findings Cha- 
racteristic for Encapsulating Peritoneal Sclerosis: A Case-Control Study. Peritoneal Dialysis International, 29, 517-522.

[8] Sampimon, D.E., Coester, A.M., Struijk, D.G. and Krediet, R.T. (2011) The Time Course of Peritoneal Transport Parameters in Peritoneal Dialysis Patients Who Develop Encapsulating Peritoneal Sclerosis. Nephrology Dialysis Transplantation, 26, 291-298. https://doi.org/10.1093/ndt/gfq343

[9] Lambie, M.L., John, B., Mushahar, L., Huckvale, C. and Davies, S.J. (2010) The Peritoneal Osmotic Conductance Is Low Well before the Diagnosis of Encapsulating Peritoneal Sclerosis Is Made. Kidney International, 78, 611-618. https://doi.org/10.1038/ki.2010.186

[10] Sampimon, D.E., Korte, M.R., Barreto, D.L., et al. (2010) Early Diagnostic Markers for Encapsulating Peritoneal Sclerosis: A Case-Control Study. Peritoneal Dialysis International, 30, 163-169. https://doi.org/10.3747/pdi.2009.00022

[11] Yamamoto, T., Nagasue, K., Okuno, S. and Yamakawa, T. (2010) The Role of Peritoneal Lavage and the Prognostic Significance of Mesothelial Cell Area in Preventing Encapsulating Peritoneal Sclerosis. Peritoneal Dialysis International, 30, 343-352. https://doi.org/10.3747/pdi.2008.00273

[12] Garosi, G. and Oreopoulos, D. (2009) No Need for an "Expiry Date" in Chronic Peritoneal Dialysis to Prevent Encapsulating Peritoneal Sclerosis. International Urology and Nephrology, 41, 903-907. https://doi.org/10.1007/s11255-009-9661-7

[13] Kawanishi, H. (2012) Surgical and Medical Treatments of Encapsulation Peritoneal Sclerosis. Contributions to Nephrology, 177, 38-47.

https://doi.org/10.1159/000336934

[14] Summers, A.M., Clancy, M.J., Syed, F., et al. (2005) Single-Center Experience of Encapsulating Peritoneal Sclerosis in Patients on Peritoneal Dialysis for End-Stage Renal Failure. Kidney International, 68, 2381-2388. https://doi.org/10.1111/j.1523-1755.2005.00701.x

[15] Moriishi, M., Kawanishi, H., Kawai, T., et al. (2002) Preservation of Peritoneal Catheter for Prevention of Encapsulating Peritoneal Sclerosis. Advances in Peritoneal Dialysis, 18, 149-153. 